\title{
Endobronchial Treatment of Carcinoid Tumors of the Lung
}

\author{
Levent Dalar ${ }^{1}$ Cengiz Ozdemir ${ }^{2}$ Yasin Abul ${ }^{3}$ Sinem Nedime Sokucu ${ }^{2}$ Levent Karasulu ${ }^{2}$ \\ Halide Nur Urer ${ }^{2}$ Sedat Altin ${ }^{2}$ \\ ${ }^{1}$ Bilim University Faculty of Medicine, Department of Pulmonary \\ Medicine, Istanbul, Turkey \\ 2 Yedikule Chest Diseases and Thoracic Surgery Teaching Hospital, \\ Istanbul, Turkey \\ ${ }^{3}$ KTU Faculty of Medicine, Department of Pulmonary Medicine, \\ Trabzon, Turkey \\ Thorac Cardiovasc Surg 2016;64:166-171. \\ Address for correspondence Yasin Abul, MD, KTU Faculty of Medicine, \\ Department of Pulmonary Medicine, Trabzon, 61080, Turkey \\ (e-mail: abulyasin@yahoo.com).
}

\begin{abstract}
Background Bronchial carcinoid tumors are known as low-grade malignancies. Surgery has been proposed as the best treatment of choice for lung carcinoids. However, less invasive treatment approaches may be considered due to low-grade malignancy potential of such tumors. The aim of this study was to review the results of endobronchial treatments of carcinoid tumors of the lung and to compare with the outcome after surgery.

Methods Initial complete tumor eradication with an endobronchial treatment was attempted for 29 patients. Diode laser or argon plasma coagulation was used during these treatments. Cryotherapy or laser treatments were applied consecutively in patients for whom there was good bronchoscopic visualization of the distal and basal tumor margins and no evidence of bronchial wall involvement. Surgery was performed in cases of atypical carcinoid and in cases of nonvisualization of the basal and distal part of the tumor.

Results Overall, 29 patients have been included (median age 58 years; range, 23-77 years). Median follow-up has been 49 months (range, 22-94 months). A total of 24 patients $(69 \%)$ had typical carcinoid tumor, 5 patients (31\%) had atypical carcinoid tumor. Initial endobronchial treatment provided complete tumor eradication in 21 of 29 patients (72\%). Of the eight other patients (28\%), two were atypical carcinoids, and underwent surgical treatment. There was no tumor-related death and no recurrence

Keywords

- carcinoid tumors

- lung

- endobronchial treatment during the follow-up in both groups. There was no difference for survival or recurrence between the surgical and the endobronchial treatment group of patients $(p>0.05)$. Conclusion Endobronchial treatment may be considered as safe, effective treatment for typical carcinoid tumors in the central airways. Addition of initial endobronchial treatment had no negative effect on the surgical outcome.
\end{abstract}

\section{Introduction}

Bronchial carcinoid tumors are rarely seen neuroendocrine tumors accounting for more than 1 to $5 \%$ of all lung tumors

received

January 10, 2015

accepted after revision

February 19, 2015

published online

May 18, 2015

and approximately 20 to $30 \%$ of all carcinoid tumors. ${ }^{1-3}$ The majority of these tumors originated from the proximal airways and most of them are symptomatic with obstructing symptoms or hemoptysis due to the tumor's hypervascularity. ${ }^{4}$ Bronchial

(c) 2016 Georg Thieme Verlag KG Stuttgart · New York
DOI http://dx.doi.org/ 10.1055/s-0035-1549274. ISSN 0171-6425. 
carcinoid tumors are mainly classified into two groups as typical and atypical bronchial carcinoids. Typical bronchial carcinoids have a good prognosis with a 10 -year survival rate of $90 \%$ compared with the atypical carcinoids that have a greater rate of metastasis and recurrence with a 10-year survival below $60 \% .{ }^{5,6}$ Generally, for patients with bronchial carcinoids surgical treatment has been the preferred approach with a 5-year survival rate of $95 \%$ after surgical treatment. ${ }^{7}$ Endobronchial treatment with laser or cryotherapy has been reported as an alternative to surgical treatment in selected patients with bronchial carcinoid tumors in individual cases. ${ }^{8-10}$ However, there have been a limited number of studies comparing endobronchial treatment with surgical treatment of bronchial carcinoids in large series, particularly with the use of cryotherapy. The aim of the present study was to review the results of endobronchial treatments including laser and cryotherapy and to compare endobronchial treatments with surgical treatment for the bronchial carcinoids.

\section{Patients and Methods}

The present study was designed as a retrospective cohort study in which all the data were retrospectively reviewed for the patients referred to the interventional pulmonology unit for bronchial carcinoid tumors between October 2006 and October 2012.

Initial complete tumor eradication by endobronchial treatment was attempted for 29 patients to improve the presurgical state with adequate tissue samples for histological classification of tumor, and to provide the minimal possible extensive parenchymal resection. Screening and staging procedures were done by contrast-enhanced computer tomography (CT) for all the study patients before any therapeutic intervention.

In studies with a retrospective analysis of standard diagnostic data, no ethical statement is needed in Turkey. Diode laser or argon plasma coagulation (APC) was used during endobronchial treatment. A diode laser operating at a wavelength in $980 \mathrm{~nm}$ with 4 to $25 \mathrm{~W}$, in a pulsed mode (Biolitec, Ceralas D 25; Jena, Germany) was used for the endobronchial treatment. APC ( $40 \mathrm{~W}$, blended mode-continuous flow) was performed using a device manufactured by ERBE Elektromedizine Gmbh, (Tübingen, Germany). Standardized protocols for appropriate power selections were used in accordance with the manufacturer's recommendations.

Cryotherapy and laser treatment was applied consecutively in patients who had complete bronchoscopic visualization of the distal and basal tumor margins and no evidence of bronchial wall involvement. Cryotherapy was performed using the ERBEKRYO system (Elektromedizine Gmbh). Treatment of lesions was performed according to the method described previously. ${ }^{11,12}$ Five cycles of freezing for 20 seconds were used for the entire tumor surface, tumor base, and a marginal area of $5 \mathrm{~mm}$ of normal mucosa around the tumor. The distance between two adjacent applications of the cryoprobe was approximately $5 \mathrm{~mm}$ providing sufficient depth of wall penetration. We performed two additional monthly cryotherapies after initial bronchoscopic excision of the tumor.
Endobronchial treatment was applied as a primary treatment modality for the patients having a strictly endoluminal disease which was previously defined ${ }^{10}$ as readily accessible to curative bronchoscopic therapy without thickening of the bronchial wall, an absence of lymph node invasion based on standard CT criteria and complete excision of the lesion if the tumor was strictly endoluminal and there was an absence of nodal enlargement.

All the patients were intubated by a rigid bronchoscope (Efer Endoscopy, La Ciotat, France) under intravenous general anesthesia using standard techniques and mechanical debridement was performed when necessary.

Bronchoscopic follow-up was done by radial probe endobronchial ultrasonography (Olympus EVIS Exera II, EU-ME1, Tokyo, Japan) and autofluorescence bronchoscopy (LIFELung, Xillix Technologies, Vancouver, Canada) after the endobronchial treatment. Surgery was performed in cases of atypical carcinoid and nonvisualization of the tumor base after endobronchial treatment and these have been called extraluminal tumor growths. The follow-up period was 49 months. The follow-up bronchoscopies were done at the $1 \mathrm{st}, 3 \mathrm{rd}, 6$ th month and at the end of the 1 st year. After the 1 year follow-up period, bronchoscopies were done every 6 months.

\section{Results}

Overall, 29 patients, 17 of them females, have been included in the study (median age 58 years; range, 23-77 years). Median follow-up was 49 months (range, 22-94 months). A total of 24 patients (69\%) had typical carcinoid tumor and 5 patients (21\%) had atypical carcinoid tumor. Initial endobronchial treatment (-Fig. 1) provided complete tumor eradication in 21 of the 29 patients ( $72 \%$ ). Of the remaining 8 of the 29 patients (28\%), 2 were atypical, and underwent surgical treatment (-Table 1). One patient who was operated for atypical carcinoid died 45 days after the operation due to sepsis. One operated case died due to ovarian carcinoma during the 45 months of follow-up period. Otherwise there was no tumor-related death and no recurrence during the follow-up period in either group of surgical treatment and/or in the group with endobronchial treatment. There was no difference in survivals or recurrences between the surgical and the endobronchial treatment groups $(p>0.05)$. Three patients in the atypical carcinoid group were given the endobronchial treatment. Symptomatic patients were 17 of the 29 patients (59\%). The most common signs and symptoms of patients with bronchial carcinoids were coughing (productive or not) in six cases (21\%), sputum production in three cases (10\%), dyspnea in ten (35\%), chest pain in five $(17 \%)$, hemoptysis in two (7\%), undefined $10 \%$. The most common symptom was dyspnea in the present study population (-Table 2).

Overall, 16 patients had right-sided bronchial carcinoid tumors and 13 patients had carcinoid tumors located in the left bronchial system.

A total of 13 patients were treated with APC as an initial endobronchial treatment. Among the 29 patients, 20 patients 


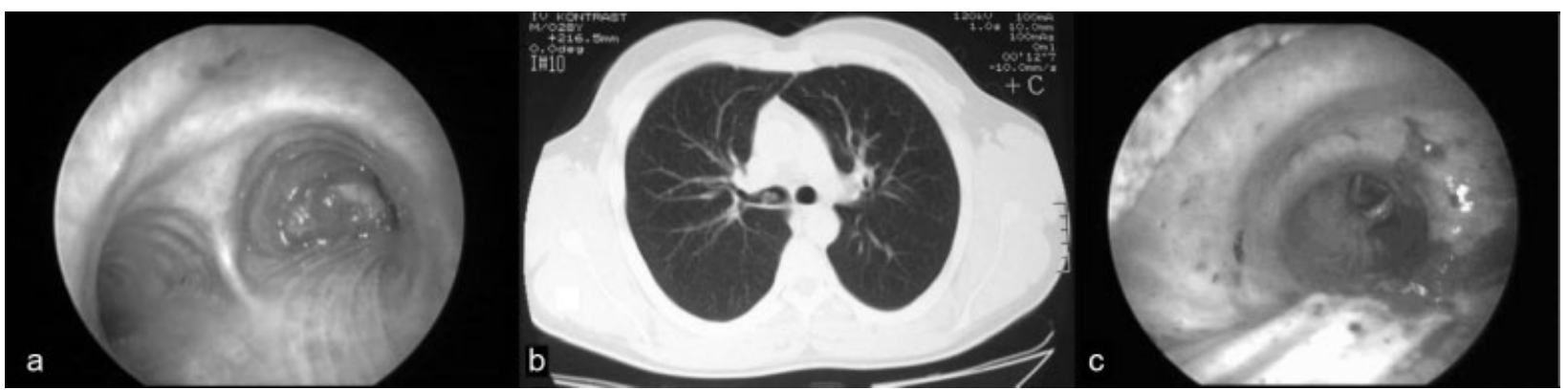

Fig. 1 (a) Endobronchial view of a typical carcinoid tumor located in right intermediate bronchus. (b) Computed tomography of the same case that shows the endobronchial tumor. (c) Bronchoscopic view of the tumor that was showed after successful endobronchial treatment (follow-up period: 64 months without recurrence).

were treated with the laser with curative intent. A total of 29 laser procedures (- Fig. 2), 18 APC procedures, 38 cryotherapy procedures, 72 rigid bronchoscopy procedures, and 86 fiberoptic bronchoscopy procedures were performed during the initial treatments and during follow-up periods of the study population. Cryotherapy was applied for 15 patients to prevent recurrence as a treatment complimentary to the initial endobronchial treatment (-Fig. 3). Two patients with bronchial carcinoids were treated solely with cryotherapy as a primary treatment modality without being any recurrence. Six patients had controlled hemorrhagic complications. There were no significant survival differences between patients treated with endobronchial treatment alone and the patients treated with surgical resection alone.

\section{Discussion}

Bronchoscopic resection of bronchial carcinoids has previously been regarded as acceptable only in patients with an inoperable condition. ${ }^{1,13}$ In the past, there seemed to be a consensus that surgical treatment of bronchial carcinoid tumors was the treatment choice for all the carcinoid tumors. ${ }^{14,15}$ However, bronchoscopic resection of bronchial carcinoid tumors has been represented as a successful modality for treatment of bronchial carcinoid tumors. ${ }^{16,17}$ Thus, at present there is no definitive consensus about endobronchial treatments of bronchial carcinoid tumors.
Actually, a decision in favor of a surgical approach has been influenced by the proportion of patients having the type of atypical carcinoids of the lung. ${ }^{18}$ The present study confirms previous studies in which selected patients with intraluminal typical bronchial carcinoids were treated by a bronchoscopic approach but also two of our patients with atypical carcinoids and with no recurrence were resected by endobronchial treatment in the present study with acceptable survival rates during follow-up period. It has previously been reported that formerly resected atypical intrapulmonary carcinoid tumors having frequent endobronchial recurrences could be effectively treated by endobronchial laser application. ${ }^{19}$ In the present study, endobronchial cryotherapy was also used as an initial sole treatment modality and as a consecutive application to prevent recurrence. Those patients primarily treated with cryotherapy and those having consecutive cryotherapy applications had no recurrence during follow-up period. Others have reported that a series of 18 patients with typical bronchial carcinoids were treated bronchoscopically by cryotherapy and during a median follow-up of 55 months, only single local recurrence $(5.5 \%)$ was noted 7 years after treatment. ${ }^{10}$ In the present study, there was no recurrence and no bronchial stenosis in the patients treated with cryotherapy during the 49 months of follow-up period. Endobronchial laser therapy has previously been reported as an effective method to treat strictly intraluminal

Table 1 Clinical characteristics of the study population

\begin{tabular}{|l|l|l|l|}
\hline Clinical feature & Total number & $\begin{array}{l}\text { APC and/or laser therapy and/or } \\
\text { cryotherapy for curative intent }\end{array}$ & $\begin{array}{l}\text { Surgery with preoperative } \\
\text { endobronchial treatment }\end{array}$ \\
\hline Number & 29 & 21 & 8 \\
\hline Sex (M:F) & $12: 17$ & $10: 11$ & $2: 6$ \\
\hline Age (median, y) & 58 & 59 & 48 \\
\hline Typical carcinoids & 24 & 19 & 5 \\
\hline Atypical carcinoids & 5 & 2 & 3 \\
\hline Bilobectomy & 2 & 2 & 2 \\
\hline Lobectomy & 4 & 4 & 4 \\
\hline Sleeve lobectomy & 2 & 1 & 2 \\
\hline
\end{tabular}

Abbreviation: APC, argon plasma coagulation. 
Table 2 Symptoms of the patients with bronchial carcinoids at the time of diagnosis

\begin{tabular}{|l|l|}
\hline Symptoms & No. (\%) \\
\hline Dyspnea & $10(35)$ \\
\hline Cough & $6(21)$ \\
\hline Chest pain & $5(17)$ \\
\hline Sputum & $3(10)$ \\
\hline Hemoptysis & $2(7)$ \\
\hline Undefined & $3(10)$ \\
\hline
\end{tabular}

typical carcinoids. ${ }^{16,17,20,21}$ Laser application was also used in the present study population as an endobronchial treatment of bronchial carcinoids. There was no recurrence during the 49 months of follow-up period in patients treated with laser application in the present study. Previous studies both with laser and cryotherapy and their bronchoscopic features are summarized in -Table 3. Accurate assessment of the tumor extension is a very important step in selecting patients with bronchial carcinoids who are candidates for endobronchial treatment. It is also important to determine the effectiveness of endobronchial treatments compared with standard treatments. Highresolution tomography, endobronchial ultrasonography, and autofluorescence bronchoscopy can be used both for assessing extension of the tumor before the treatment and for the determining effectiveness of endobronchial treatment during follow-up. ${ }^{9,19}$ High-resolution tomography, endobronchial ultrasonography, and autofluorescence bronchoscopy (-Fig. 4) were used in the present study to follow-up the study population. Lung carcinoid tumors have been usually described as tumors with low grade of fluorodeoxyglucose (FDG) uptake due to tumors' low metabolism and slow growth. Therefore, FDG-positron emission tomography (PET) has been known to have a reduced sensitivity for carcinoids and a limited role in the diagnosis of those lesions. Some controversial results have been proposed on the diagnostic accuracy of FDG-PET for bronchial carcinoids. However, there is some evidence reporting the use of PET with analogues of somatostatin including DOTA-TOC, DOTA-NOC, or DOTA-TATE labeled with gallium-68 (Ga-68) in pulmonary carcinoids. ${ }^{22}$ Ga68-DOTATOC PET/CT is a useful thoracic imaging technique to evaluate pulmonary carcinoids with sensitivity of 96 and $100 \%$ specific whereas $18 \mathrm{~F}-\mathrm{FDG}$ PET/CT scan provides low sensitivity and specificity to differentiate pulmonary carcinoids from other lung malignancies. ${ }^{23}$ Magnetic resonance imaging (MRI) usually does not provide more efficient knowledge than CT, but dynamic contrast-enhanced MRI may be useful to differentiate peripheral carcinoids that have small contrast-enhancement from pulmonary vessels, or to define vascular involvement by a central lesion. ${ }^{24}$ When compared with the pathological size of the lesion, endobronchial ultrasound measurements were reported as more accurate than high-resolution CT measurements. ${ }^{25}$

Although bronchial carcinoids have a low potential for malignancy, posttreatment surveillance of patients with bronchial carcinoid tumors is necessary because of the recurrence potential of the tumors after initial treatment. The optimal posttreatment surveillance strategy is not well defined, and there is no consensus about follow-up of bronchial carcinoids. $^{26}$

The main limitation of the present study is its retrospective design. The second limitation is that the present study is not a randomized controlled study and the third is the fact that its conclusions are based on a single center experience. Although the study includes moderate number of patients for such a rare tumor, it could be considered that the power of the present study is limited. Studies comparing endobronchial treatment with surgical treatment are limited in the literature. ${ }^{21}$ Long-term outcomes of bronchoscopically resected endobronchial typical carcinoid tumors has been reported as acceptable. $^{20,27,28}$ There were no significant differences between the patients receiving endobronchial treatment and patients receiving surgical therapy in the present study. However, the study group of the patients with surgical treatment is limited in number. It should be stressed that treatment of two atypical bronchial carcinoids with endobronchial treatment without any recurrence is important from the point of using of endobronchial treatment in selected bronchial carcinoids.

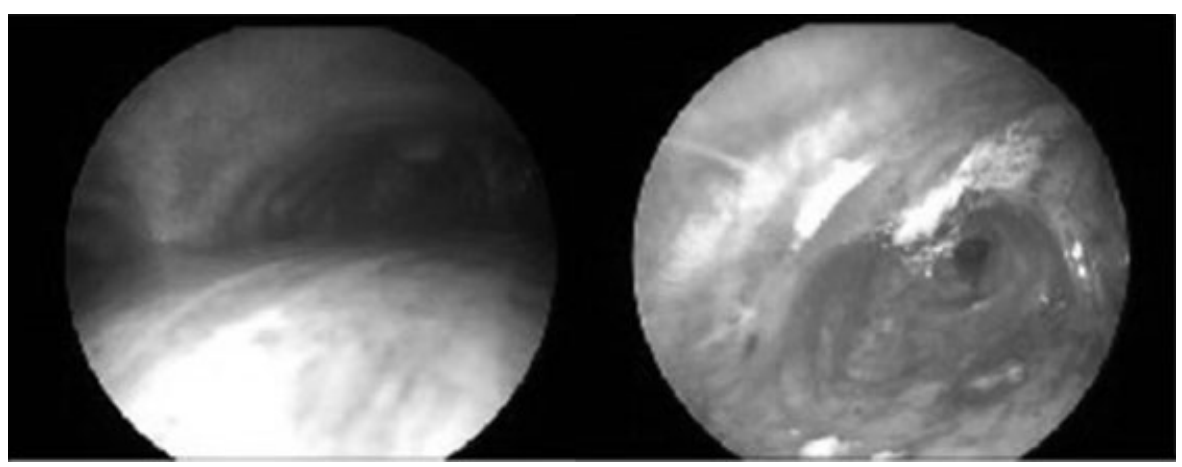

Fig. 2 A 53-year-old male patient had a history of bronchoscopic resection of carcinoid tumors located in the intermediate bronchus. Minimal residual tissue and view of laser ablation are seen. Follow-up period is 67 months without any recurrence. 


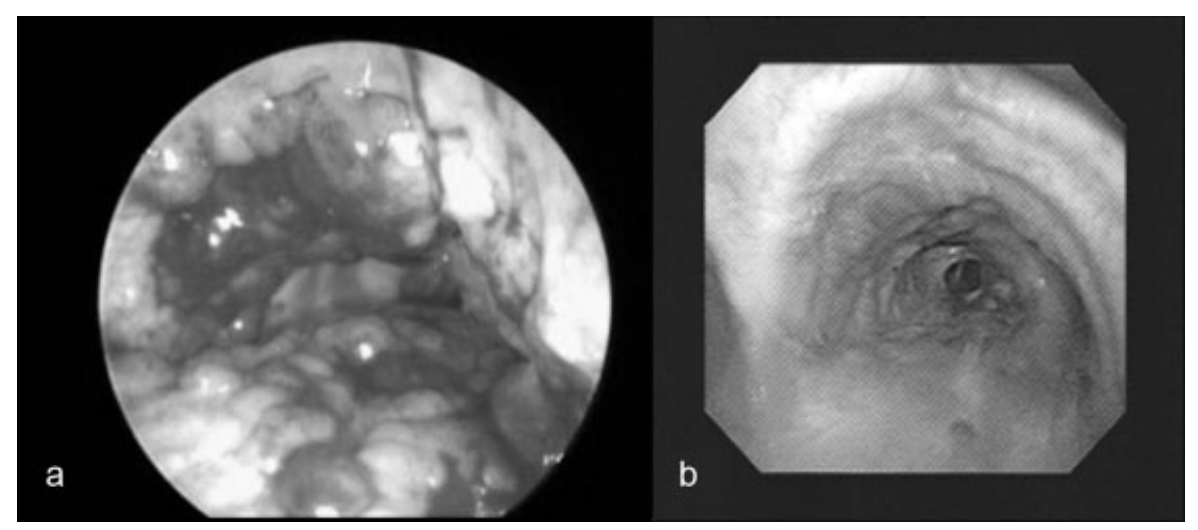

Fig. 3 (a) Atypical bronchial carcinoids within right main bronchus, showing the widespread nodularity within the proximal part of the right main bronchus. (b) Endobronchial view of the lesion is seen after 1 year consecutive cryotherapy.

Table 3 Literature studies including endobronchial treatment of bronchial carcinoids with curative intent

\begin{tabular}{|l|l|l|l|l|}
\hline Clinical studies & $\begin{array}{l}\text { Number of study } \\
\text { population for endobronchial } \\
\text { treatment }\end{array}$ & $\begin{array}{l}\text { Follow-up time } \\
\text { (median/mo) }\end{array}$ & Cure rate (\%) & Bronchoscopic technique \\
\hline Sutedja et al & 11 & 70 & 55 & RB + laser or mechanical under GA \\
\hline Cavaliere et al & 38 & 24 & 92 & RB + laser under GA \\
\hline van Boxem et al & 19 & 29 & 73 & RB + laser under GA \\
\hline Luckraz et al & 28 & 105 & 94 & RB + MR under GA \\
\hline Bertoletti et a & 18 & 55 & 96 & $\begin{array}{l}\text { RB + cryotherapy under GA } \\
\text { (14 patients) }\end{array}$ \\
\hline Fuks et al & 10 & 24 & 100 & FB + cryotherapy (4 patients) \\
\hline Neyman et al & 25 & 36 & 94 & RB + laser under GA \\
\hline Brokx et al & 33 & 65 & 100 & $\begin{array}{l}\text { RB + FB + Nd:YAG } \\
\text { laser or electrocautery under GA }\end{array}$ \\
\hline The present study & 29 & 49 & 100 & RB + laser \pm cryotherapy under GA \\
\hline
\end{tabular}

Abbreviations: CS, conscious sedation; FB, flexible bronchoscopy; GA, general anesthesia; MR, mechanical resection; RB, rigid bronchoscopy.

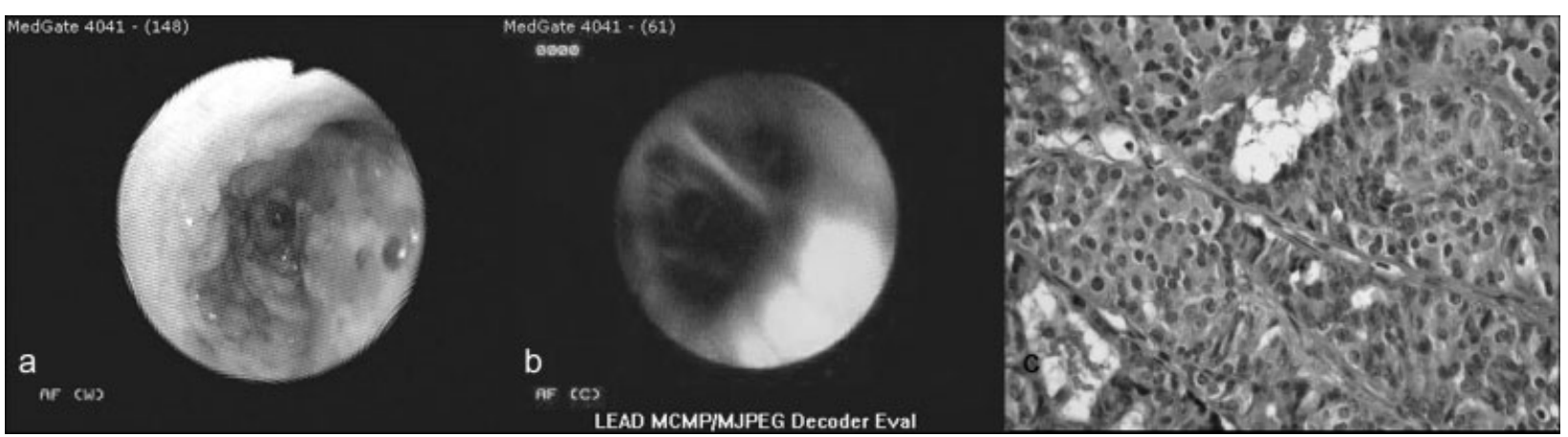

Fig. 4 (a) Endobronchial view of atypical carcinoids with nodularity located in right lower lobe bronchus is seen. (b) Autofluorescence bronchoscopic view of the same lesion is demonstrated. (c) Neuroendocrine tumor cells that shows nuclear atypical pattern. Hematoxylin and eosin stain, $\times 400$.

\section{Conclusions}

Endobronchial treatment is a potentially more tissue-protecting alternative than early surgical resection in patients with intraluminally located carcinoids. The current study has a retrospective design and no randomization has been done. In spite of these limitations, endobronchial laser treatment and cryotherapy may be considered as safe and effective treatment modalities in the treatment of carcinoid tumors in the central airways with intraluminal tumor growth. Addition of initial endobronchial treatment had no negative effect on surgical treatment outcome. 


\section{Acknowledgment}

The authors thank Prof. R. W. Guillery from Oxford University for the correction of English in the article.

\section{References}

1 Davila DG, Dunn WF, Tazelaar HD, Pairolero PC. Bronchial carcinoid tumors. Mayo Clin Proc 1993;68(8):795-803

2 Modlin IM, Lye KD, Kidd M. A 5-decade analysis of 13,715 carcinoid tumors. Cancer 2003;97(4):934-959

3 Hemminki K, Li X. Incidence trends and risk factors of carcinoid tumors: a nationwide epidemiologic study from Sweden. Cancer 2001;92(8):2204-2210

4 Skuladottir H, Hirsch FR, Hansen HH, Olsen JH. Pulmonary neuroendocrine tumors: incidence and prognosis of histological subtypes. A population-based study in Denmark. Lung Cancer 2002; 37(2):127-135

5 Mezzetti M, Raveglia F, Panigalli T, et al. Assessment of outcomes in typical and atypical carcinoids according to latest WHO classification. Ann Thorac Surg 2003;76(6):1838-1842

6 Travis WD, Rush W, Flieder DB, et al. Survival analysis of 200 pulmonary neuroendocrine tumors with clarification of criteria for atypical carcinoid and its separation from typical carcinoid. Am J Surg Pathol 1998;22(8):934-944

7 Rizzardi G, Marulli G, Bortolotti L, Calabrese F, Sartori F, Rea F. Sleeve resections and bronchoplastic procedures in typical central carcinoid tumours. Thorac Cardiovasc Surg 2008;56(1):42-45

8 Brokx HA, Risse EK, Paul MA, et al. Initial bronchoscopic treatment for patients with intraluminal bronchial carcinoids. J Thorac Cardiovasc Surg 2007;133(4):973-978

9 van Boxem TJ, Golding RP, Venmans BJ, Postmus PE, Sutedja TG. High-resolution CT in patients with intraluminal typical bronchial carcinoid tumors treated with bronchoscopic therapy. Chest 2000; 117(1):125-128

10 Bertoletti L, Elleuch R, Kaczmarek D, Jean-François R, Vergnon JM. Bronchoscopic cryotherapy treatment of isolated endoluminal typical carcinoid tumor. Chest 2006;130(5):1405-1411

11 Deygas N, Froudarakis M, Ozenne G, Vergnon JM. Cryotherapy in early superficial bronchogenic carcinoma. Chest 2001;120(1):26-31

12 Vergnon JM. Bronchoscopic cryotherapy. J Bronchol 1995; 2:323-327

13 Díaz-Jiménez JP, Canela-Cardona M, Maestre-Alcacer J. Nd:YAG laser photoresection of low-grade malignant tumors of the tracheobronchial tree. Chest 1990;97(4):920-922

14 Okike N, Bernatz PE, Payne WS, Woolner LB, Leonard PF. Bronchoplastic procedures in the treatment of carcinoid tumors of the tracheobronchial tree. J Thorac Cardiovasc Surg 1978;76(3): 281-291

15 Aberg T, Blöndal T, Nõu E, Malmaeus J. The choice of operation for bronchial carcinoids. Ann Thorac Surg 1981;32(1):19-22

16 Sutedja TG, Schreurs AJ, Vanderschueren RG, Kwa B, vd Werf TS, Postmus PE. Bronchoscopic therapy in patients with intraluminal typical bronchial carcinoid. Chest 1995;107(2):556-558

17 van Boxem TJ, Venmans BJ, van Mourik JC, Postmus PE, Sutedja TG. Bronchoscopic treatment of intraluminal typical carcinoid: a pilot study. J Thorac Cardiovasc Surg 1998;116(3):402-406

18 McCaughan BC, Martini N, Bains MS. Bronchial carcinoids. Review of 124 cases. J Thorac Cardiovasc Surg 1985;89(1):8-17

19 Katsenos S, Rojas-Solano J, Schuhmann M, Becker HD. Bronchoscopic long-term palliation of a recurrent atypical carcinoid tumor. Respiration 2011;81(4):345-350

20 Fuks L, Fruchter O, Amital A, Fox BD, Abdel Rahman N, Kramer MR. Long-term follow-up of flexible bronchoscopic treatment for bronchial carcinoids with curative intent. Diagn Ther Endosc 2009;2009:782961

21 Neyman K, Sundset A, Naalsund A, et al. Endoscopic treatment of bronchial carcinoids in comparison to surgical resection: a retrospective study. J Bronchology Interv Pulmonol 2012; 19(1):29-34

22 Lococo F, Cesario A, Paci M, et al. PET/CT assessment of neuroendocrine tumors of the lung with special emphasis on bronchial carcinoids. Tumour Biol 2014;35(9):8369-8377

23 Venkitaraman B, Karunanithi S, Kumar A, Khilnani GC, Kumar R. Role of 68Ga-DOTATOC PET/CT in initial evaluation of patients with suspected bronchopulmonary carcinoid. Eur J Nucl Med Mol Imaging 2014;41(5):856-864

24 Douek PC, Simoni L, Revel D, Cordier JF, Amiel M. Diagnosis of bronchial carcinoid tumor by ultrafast contrast-enhanced MR imaging. AJR Am J Roentgenol 1994;163(3):563-564

25 Bostanci K, Solano JR, Becker HD. Is there a role of EBUS in the decision-making of endoscopic treatment for carcinoid tumors? Eur Respir J 2011;38(55):231

26 Phan AT, Oberg K, Choi J, et al; North American Neuroendocrine Tumor Society (NANETS). NANETS consensus guideline for the diagnosis and management of neuroendocrine tumors: welldifferentiated neuroendocrine tumors of the thorax (includes lung and thymus). Pancreas 2010;39(6):784-798

27 Luckraz H, Amer K, Thomas L, Gibbs A, Butchart EG. Long-term outcome of bronchoscopically resected endobronchial typical carcinoid tumors. J Thorac Cardiovasc Surg 2006;132(1):113-115

28 Cavaliere S, Foccoli P, Toninelli C. Curative bronchoscopic laser therapy for surgically resectable tracheobronchial tumors: personal experience. Journal of Bronchology 2002;9(2):90-95 